\title{
Análisis numérico de la respuesta sísmica de excavaciones sostenidas con muro Berlinés en arena
}

\author{
Numerical analysis of the seismic response of excavations supported by soldier pile walls in \\ sand \\ Fecha de entrega: 5 de noviembre 2014 \\ Fecha de aceptación: 20 de abril 2015
}

\section{Mauricio Jara ${ }^{1}$, Felipe Villalobos ${ }^{2}$, Gonzalo Corral $^{3}$ y Óscar Taiba ${ }^{4}$}

${ }^{1}$ Macrosteel - Soluciones Integrales de Ingeniería, Chacabuco 485, 5to piso, Concepción, Chile, mjara@macrosteel.cl
2 Laboratorio de GeoMateriales, Universidad Católica de la Santísima Concepción, Alonso de Ribera 2850, Casilla 297,
Concepción, Chile, avillalobos@ucsc.cl
${ }^{3}$ ITASCA S.A., Dr. José Luis Aguilar 1178, Providencia, Santiago, Chile, gonzalo.corral@itasca.cl
${ }^{4}$ Ferrara - Proyectos Especiales, Rengo 1270, Nuñoa, Santiago, Chile, oscartaiba@ferrara.cl

Se presenta un análisis de respuesta sísmica de excavaciones usando un programa comercial de elementos finitos que considera la interacción suelo-muro y el efecto de las etapas constructivas en arena Bío Bío durante el evento sísmico del 27F/2010. Los resultados permiten concluir que puntos ubicados en el perímetro superior de la excavación pueden alcanzar valores de aceleración artificial máxima cercanos a $0.52 g$ y factores de amplificación cercanos a 10, con desplazamientos horizontales relativos mayores a $20 \mathrm{~mm}$. Los espectros de respuesta artificiales obtenidos muestran variaciones pequeñas en el periodo fundamental del sistema hasta una profundidad de $12 \mathrm{~m}$. Finalmente, el uso del parámetro $A_{r}$ recomendado por la normativa nacional NCh3206 (2010) resulta razonable para excavaciones no superiores a $5 \mathrm{~m}$ y para aquellas que superan los $10 \mathrm{~m}$ el efecto destructivo del sismo se ve incrementado por el contexto geotécnico local y perturbaciones del sistema analizado.

Palabras clave: respuesta sísmica, muro Berlinés, elementos finitos, excavación, arena Bío Bío
A seismic response analysis of an excavation using a finite elements commercial program which considers the soil-wall interaction and the construction sequence effect, is presented for Bio Bio sand during the 27F/2010 earthquake. Results allow the conclusion that points around the excavation surface perimeter can reach maximum artificial acceleration values close to $0.52 \mathrm{~g}$ and amplification factors close to 10, with relative horizontal displacements larger than $20 \mathrm{~mm}$. The artificial response spectra obtained show small variations of the system fundamental period up to a depth of $12 \mathrm{~m}$. Finally, it seems reasonable the use of the $A_{r}$ parameter recommended by the national norm NCh3206 (2010) for excavation depths not deeper than $5 \mathrm{~m}$. However, for excavations deeper than $10 \mathrm{~m}$ the seismic damage can increase due to the local geotechnical conditions and perturbations of the analyzed system.

Keywords: seismic response, soldier pile wall, finite elements, excavation, Bío Bío sand

\section{Introducción}

En la actualidad se desarrollan cada vez más proyectos que requieren de excavaciones profundas en medio de las ciudades. Lo anterior implica que tanto el diseño y construcción se vuelven aún más complejos por lo restringido del espacio de trabajo y por las altas demandas de seguridad de las estructuras aledañas. El presente trabajo se enfoca en el análisis de excavaciones sostenidas con muros Berlinés anclados en suelos arenosos de la ciudad de Concepción. Las recomendaciones nacionales actuales (NCh3206, 2010) proponen evaluar la acción sísmica sobre estructuras de retención flexibles a través de empujes uniformes directamente proporcionales a la máxima aceleración del suelo $A_{\mathrm{o}}$. Esta demanda puede ser reducida a una aceleración $A_{\mathrm{r}}$ en función del tipo de suelo y de la magnitud admitida para el desplazamiento post sismo del muro. La interacción sísmica suelo-muro asociada a los desplazamientos, es abordada de manera indirecta mediante 
el factor de reducción $\alpha$, que se obtiene aplicando la metodología del bloque deslizante propuesta por Newmark (1965). El empleo de estas recomendaciones ha tenido un comportamiento aceptable, pero a su vez tiende a omitir factores propios de la zona de estudio como amplificación dinámica, etapas constructivas y desplazamientos acumulados. Mayormente para el análisis de estos casos se utilizan métodos pseudo-estáticos de equilibrio límite, los cuales no permiten estimar campos de deformaciones ni de tensiones del suelo alrededor de la excavación, de ahí surge la necesidad de usar métodos numéricos, que permitan evaluar de mejor manera la interacción dinámica entre una estructura y el suelo. Este trabajo propone investigar mediante análisis dinámicos el comportamiento sísmico de excavaciones con profundidades superiores a $10 \mathrm{~m}$.

\section{Metodología}

Se elabora un modelo numérico basado en el método de elementos finitos MEF implementado en el programa computacional comercial Plaxis V8.2 (2010), utilizando las propiedades mecánicas y dinámicas de la arena Bío Bío. El registro de aceleraciones utilizado a nivel basal del modelo, es el del 27F/2010 en afloramiento rocoso de la Universidad Técnica Federico Santa María UTFSM, su elección obedece a la no existencia de un registro en roca en Concepción y resultados mediante el uso de deconvolución no satisfactorios para los registros superficiales del mismo lugar. Se estima la respuesta artificial en el contorno de la excavación a través de puntos de control para cada etapa de construcción. La Tabla 1 presenta datos usados en el análisis correspondiente a cada estrato modelado. Estos datos fueron obtenidos principalmente a partir de una base de datos de sondajes realizados en el centro de la ciudad y ensayos de laboratorio (Mozó, 2012; Mozó et al., 2012). También se utilizó una distribución de velocidades de ondas de corte $V_{\mathrm{s}}$ en función de la profundidad, correspondiente a un ajuste para mediciones realizadas con bender elements en arena Bío Bío (Ayala, 2013). Dicho ajuste es mostrado en la Figura 1 y su extrapolación para el nivel tensional correspondiente al depósito es presentado en la Figura 2. El basamento rocoso está compuesto principalmente por roca granítica, con profundidades variables dependiendo del sector de la ciudad, rango de profundidades obtenido mediante métodos geoeléctricos con verificaciones de sondajes mecánicos superficiales que va desde 0 a $130 \mathrm{~m}$ (Gutiérrez, 1991).

Se utilizó el modelo constitutivo Hardening Soil para el comportamiento de la arena, el cual se enmarca dentro de la teoría de plasticidad de endurecimiento por deformación de corte, que incluye el endurecimiento por compresión (Plaxis V8.2, 2010; Shanz et al., 1999). Se asumirá además un análisis no drenado que represente la generación de presión de poros. En relación a la condición de campo libre el modelo considera bordes absorbentes, una interfaz

Tabla 1: Valores de los parámetros usados en los análisis numéricos

\begin{tabular}{|c|c|c|c|c|c|c|c|c|c|c|}
\hline Parámetro & H1 & H 2 & H 3 & H 4 & H 5 & H 6 & $\mathrm{H} 7$ & H 8 & H 9 & H 10 \\
\hline Profundidad $z, m$ & 3.5 & 5.5 & 8.0 & 25.0 & 28.0 & 50.0 & 85.0 & 100.0 & 130.0 & $>130$ \\
\hline Espesor $H, \mathrm{~m}$ & 3.5 & 2.0 & 2.5 & 17.0 & 3.0 & 22.0 & 35.0 & 15.0 & 30.0 & IND \\
\hline Modelo del material & HS & HS & HS & HS & HS & HS & HS & HS & HS & LE \\
\hline Comportamiento del material & ND & ND & ND & ND & ND & ND & ND & ND & ND & NP \\
\hline Peso unitario sumergido $\gamma^{\prime}, \mathrm{kN} / \mathrm{m}^{3}$ & 8.2 & 9.2 & 10 & 10 & 10 & 10 & 10 & 10 & 10 & 26.5 \\
\hline Peso unitario $\gamma, \mathrm{kN} / \mathrm{m}^{3}$ & 18 & 19 & 20 & 20 & 20 & 20 & 20 & 20 & 20 & 26.5 \\
\hline Clasificación USCS & SM & SM & SP & SP & SP & SP & SP & $\mathrm{SP}$ & SP & RG \\
\hline Módulo elástico $E, \mathrm{MPa}$ & 161 & 199 & 239 & 349 & 361 & 418 & 493 & 519 & 563 & 55917 \\
\hline Módulo secante triaxial $E_{50}, \mathrm{MPa}$ & 194 & 238 & 287 & 419 & 434 & 464 & 548 & 577 & 626 & - \\
\hline Módulo edométrico tangente $E_{\mathrm{ed}}, \mathrm{MPa}$ & 194 & 238 & 287 & 419 & 434 & 464 & 548 & 577 & 626 & - \\
\hline Módulo carga/descarga $E_{\text {ur }}, \mathrm{MPa}$ & 581 & 715 & 860 & 1257 & 1303 & 1393 & 1645 & 1731 & 1879 & - \\
\hline Ángulo de fricción $\phi,^{\circ}$ & 33 & 37 & 42 & 42 & 42 & 42 & 42 & 42 & 42 & $>45$ \\
\hline Relación de Poisson $v$ & 0.25 & 0.25 & 0.25 & 0.25 & 0.25 & 0.20 & 0.20 & 0.20 & 0.20 & 0.15 \\
\hline Módulo de corte máximo $G_{\max }, M P a$ & 65 & 79 & 96 & 140 & 145 & 174 & 206 & 216 & 235 & 24312 \\
\hline Velocidad de ondas de corte $V_{\mathrm{s}}, \mathrm{m} / \mathrm{s}$ & 188 & 203 & 217 & 262 & 266 & 292 & 318 & 326 & 339 & $>3000$ \\
\hline Velocidad de ondas de compresión $V_{p}, \mathrm{~m} / \mathrm{s}$ & 325 & 351 & 375 & 453 & 462 & 477 & 519 & 532 & 554 & $>4675$ \\
\hline Coeficiente de permeabilidad $k, \mathrm{~m} / \mathrm{s}$ & 1.4E-03 & $1.4 \mathrm{E}-03$ & 8.0E-04 & 8.0E-04 & 8.0E-04 & 8.0E-04 & 8.0E-04 & 8.0E-04 & 8.0E-04 & $1.0 \mathrm{E}-10$ \\
\hline Factor de reducción de resistencia $R_{\text {inter }}$ & 0.67 & 0.67 & 0.80 & 0.80 & 0.80 & 0.80 & 0.80 & 0.80 & 0.80 & 一 \\
\hline
\end{tabular}

HS Hardening Soil, LE Lineal Elástico, ND No Drenado, NP No Poroso, IND Indefinido, RG Roca Granítica 
entre el suelo y la estructura permite la compatibilidad de desplazamientos y además conserva una relación de aspecto máxima ancho/altura $(260 \mathrm{~m} / 130 \mathrm{~m}$ ) igual a 2 (Figura 3). Esto permite desestimar reducciones de amplitudes para altas frecuencias y amplificaciones de estas mismas para frecuencias bajas (sismo) provocadas por relaciones mayores a 2, lo anterior en función de lo propuesto por Sharahi (2010) y Molina et al. (2008).

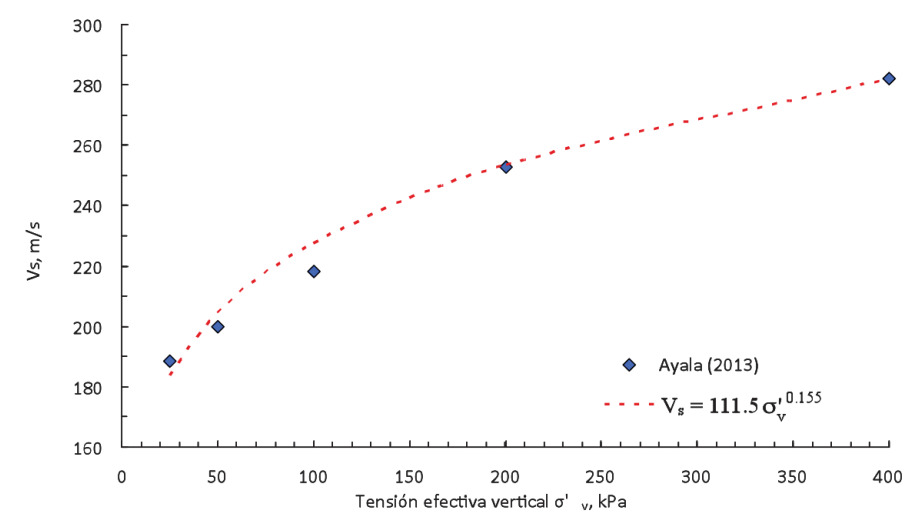

Figura 1: Ajuste de $V_{\mathrm{s}}$ versus tensión efectiva en arena Bío Bío con datos de ensayos con bender elements (Ayala, 2013)

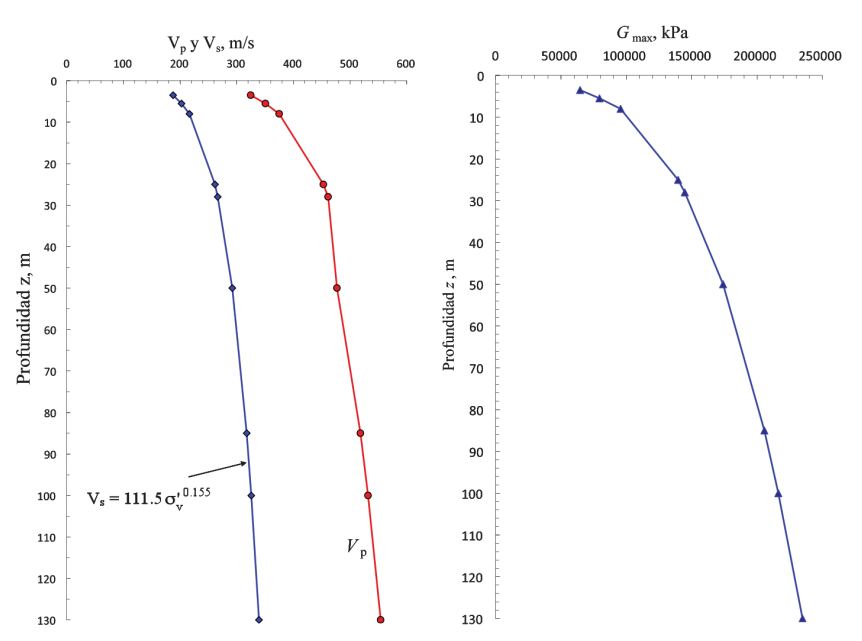

Figura 2: Variación en profundidad de a) $V_{\mathrm{s}}$ y $V_{\mathrm{p}}$ y b) del módulo de corte $G_{\max }$

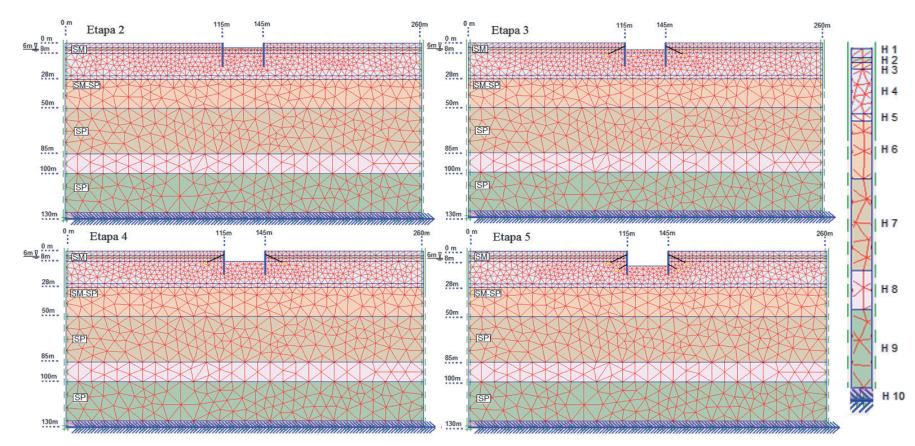

Figura 3: Etapas constructivas y sistema de elementos finitos utilizado
El modelo consta de 5 etapas, siendo la primera de ellas considerada sin retiro de material y a su vez como parámetro inicial de comparación; mientras que para las etapas 2, 3, 4 y 5 las profundidades de excavación son 3.5, 5.5, 8.0 y $12 \mathrm{~m}$ respectivamente (Figura 3). El espesor del muro Berlinés considerado es de $0.5 \mathrm{~m}$, con rigideces en el orden de $5.94 \cdot 10^{6} \mathrm{kN} / \mathrm{m}$ (EA) y $0.13 \cdot 10^{6} \mathrm{kNm}^{2} / \mathrm{m}$ (EI), la longitud de embebido es calculada para la etapa 5 ( $z \geq$ $12 \mathrm{~m}$ ) según resoluciones analíticas y de equilibrio límite (Kranz, 1953), el valor obtenido es de $6 \mathrm{~m}$ implicando una longitud total de $18 \mathrm{~m}$ para el muro. Las zonas deslizantes del suelo detrás del muro indican que para realizar la etapa 3 de excavación $(z \geq 3.5 \mathrm{~m})$ el sistema necesita una primera línea de anclajes a una profundidad equivalente a $2 / 3$ de la profundidad de excavación de la etapa 2 (2.33 $\mathrm{m})$, con un ángulo de $23^{\circ}$ con respecto a la horizontal del muro. Para la etapa 5 es necesaria una segunda línea de anclajes, la cual se dispone a una profundidad igual a $8 \mathrm{~m}$ con una inclinación idéntica a la primera línea. La longitud de los anclajes es en función de la extensión de la cuña deslizante, siendo de $14.5 \mathrm{~m}$ para la primera y $10 \mathrm{~m}$ para la segunda línea (Figura 4). Además la Figura 4 muestra los puntos de control para cada etapa de construcción (de A a J). Para estos análisis dinámicos se consideró simetría en la excavación, el nivel freático ubicado a $6 \mathrm{~m}$ de profundidad y además se desestimó la incorporación de cargas de superestructuras vecinas por considerarse un factor que haría aún más complejo la interpretación de resultados. Investigaciones posteriores tienen contemplado incorporar sobrecargas en el análisis.
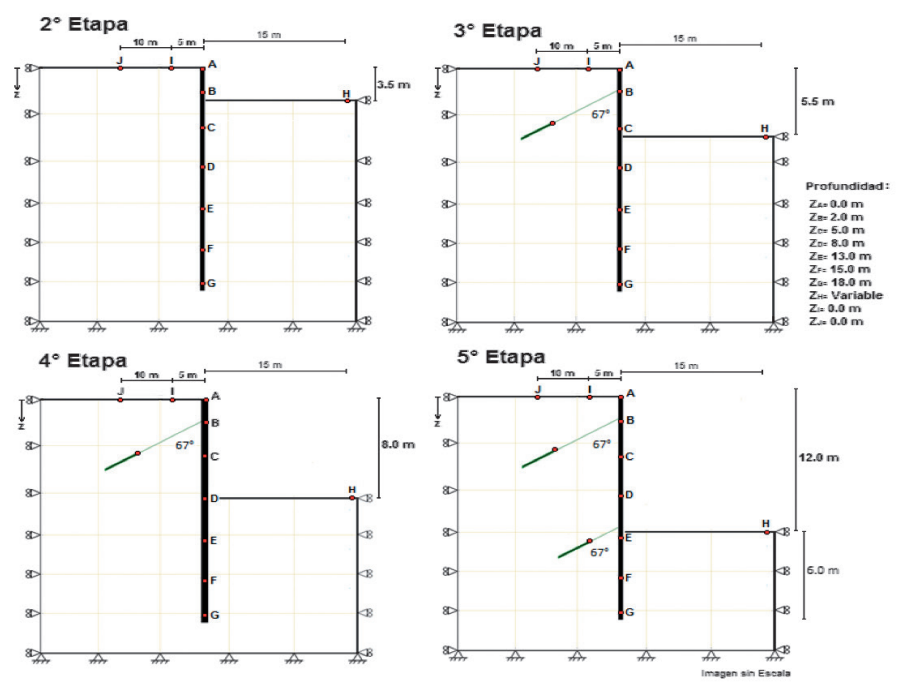

Figura 4: Ubicación de los puntos de control y etapas de excavación (sistema simétrico) 
Se siguieron las recomendaciones de Kuhlmeyer y Lysmer (1973) para acotar el tamaño máximo de los elementos finitos $\left(L_{\max } \leq \lambda / 5\right)$ en función de la longitud de las ondas de corte y la frecuencia más representativa de la onda incidente $\left(\lambda \leq V_{\mathrm{s}} / f\right)$, esto para evitar la distorsión numérica del paso de las ondas a través del modelo. Se utilizará un valor aproximado para $f$ igual a $6.25 \mathrm{~Hz}$ (ver Figura 5c) y un valor de $V_{\mathrm{s}}$ representativo para el depósito igual a $304 \mathrm{~m} / \mathrm{s}$, se sigue que el valor de longitud máxima para los elementos finitos asociado a estos parámetros es $9.8 \mathrm{~m}$. La onda incidente utilizada será la componente transversal del registro de aceleraciones en afloramiento rocoso medido en la estación UTFSM correspondiente al terremoto 27F/2010. En la Figura 5 es mostrado el registro de aceleraciones y el espectro respuesta de pseudoaceleraciones y de Fourier correspondiente para 5\% de amortiguamiento, donde el amortiguamiento natural es de tipo histerético e independiente de la frecuencia. Para este análisis se ha utilizado un amortiguamiento mecánico del tipo Rayleigh, en donde la matriz de amortiguamiento es función de parámetros proporcionales a las matrices de masa y rigidez. La elección de estos parámetros fue realizada de modo de cubrir el rango de frecuencias más importante de la onda incidente utilizada, en este caso el rango de periodos más importante de la respuesta espectral para una razón de amortiguamiento de $5 \%$ está entre 0.1 y $0.5 \mathrm{~s}$ (ver Figura 5b).

\section{Resultados del análisis}

\section{Aceleraciones artificiales}

En la Figura 6a se compara el registro artificial en el punto A para el caso sin excavación y en el mismo punto para el caso de excavación en la 5ta etapa. El PGA para el caso sin excavación es de $0.43 \mathrm{~g}$ mientras que para el caso con excavación sube a $0.52 \mathrm{~g}$, indicando un aumento de un $20 \%$ en la demanda sísmica y además superando la aceleración máxima $A_{\mathrm{o}}$ en un $30 \%$. La Figura $6 \mathrm{~b}$ muestra la diferencia de aceleraciones entre los dos registros ( $\sin$ y con excavación), donde la mayor diferencia está en el rango de tiempo entre 40 hasta $48 \mathrm{~s}$.

Las aceleraciones máximas estimadas en cada punto de control sobre el muro son mostrados en la Figura 7 para cada etapa de excavación. Se observa que la aceleración máxima estimada en superficie en cada etapa constructiva
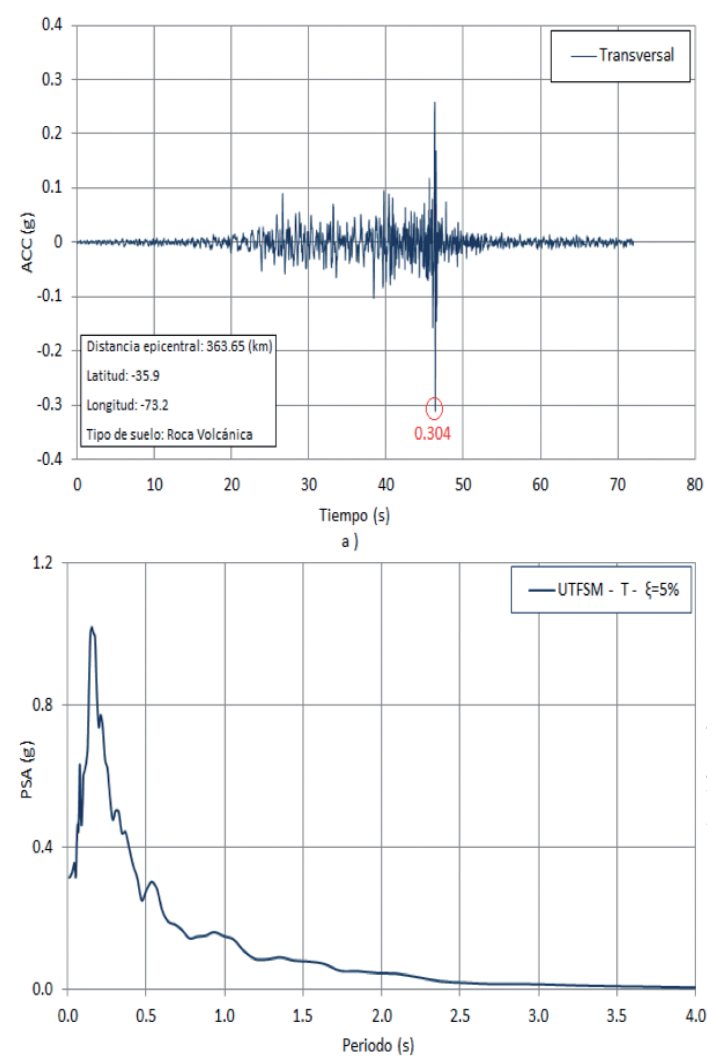

b)

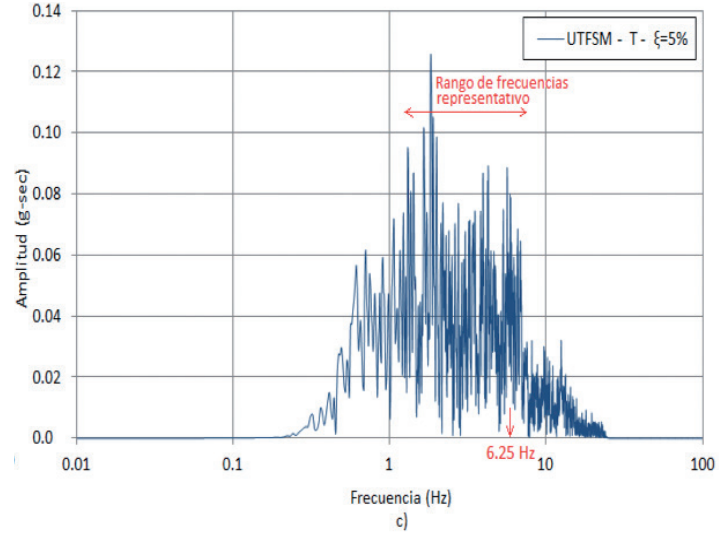

Figura 5: a) Registro de aceleraciones, b) pseudo-espectro de respuesta y c) espectro de Fourier, estación UTFSM dirección transversal ( $5 \%$ de amortiguamiento)

duplica a la aceleración reducida $A_{\mathrm{r}}$ y además superan en un $16 \%$ promedio la aceleración máxima sin excavación. Los resultados confirman de manera preliminar que la acción de un sismo sobre una excavación sostenida por un sistema flexible embebido, con una profundidad superior a los $8 \mathrm{~m}$, podría tener incrementos en las aceleraciones en superficie, no cubiertas por las recomendaciones de cálculo establecidas. El fenómeno de amplificación de la aceleración es atribuible al cambio de geometría, la 
excavación reduce la rigidez del suelo y además perturba la llegada de las ondas de corte, provocando que los fenómenos de impedancia y resonancia amplifiquen la respuesta en los contornos de la excavación. Por otro lado, el valor de $0.5 \mathrm{~g}$ para el punto A se mantiene casi constante para todas las etapas, mientras que en los puntos I y J la aceleración tiende a aumentar con la profundidad, indicando el efecto de la excavación sobre la vecindad.

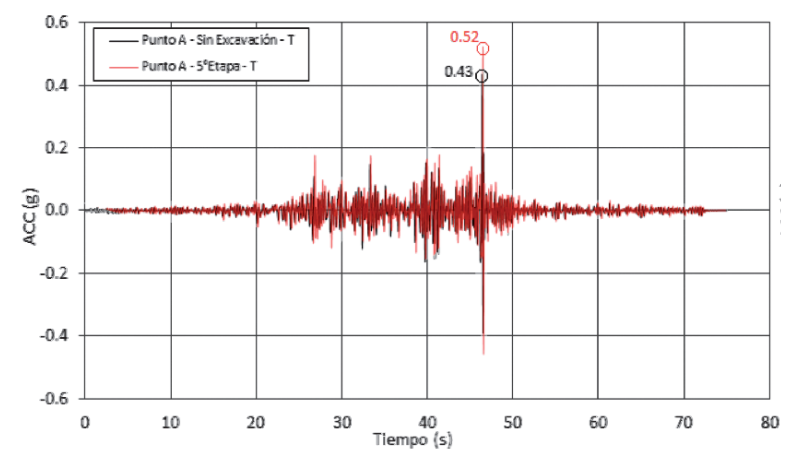

(a)

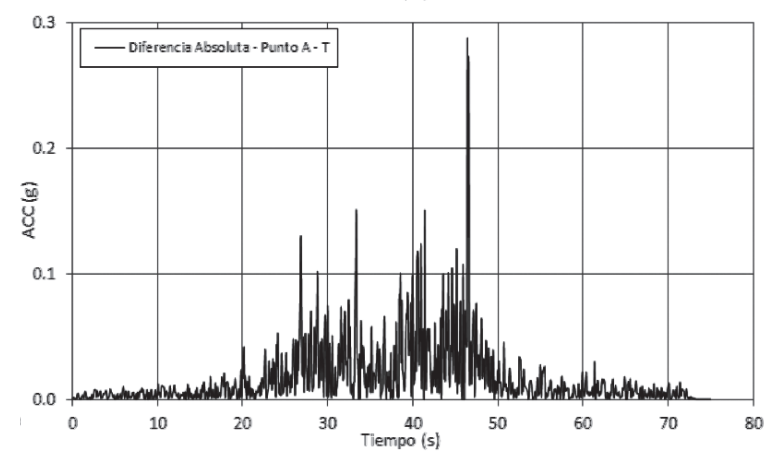

(b)

Figura 6: a) Comparación de registros artificiales en el punto A, y b) diferencia absoluta de los registros obtenidos en el punto A

\section{Respuesta espectral}

La Figura 8 muestra la respuesta espectral para cada etapa constructiva, donde es posible notar que no existe una variación importante del periodo fundamental hasta el punto $\mathrm{D}$, siendo el periodo en promedio igual a $0.17 \mathrm{~s}$. Para la parte del muro empotrado sea puntos más profundos el periodo tiende a aumentar, esto debido probablemente a un efecto de vibración libre en la punta de los perfiles del muro. Por otro lado, la amplitud del pseudo-espectro de aceleración PSA tiende a aumentar con el volumen de material excavado, lo que es asociado a que al incrementar el volumen potencialmente deslizante crece también la masa sísmica, aumentado la respuesta para los puntos más cercanos a la superficie.
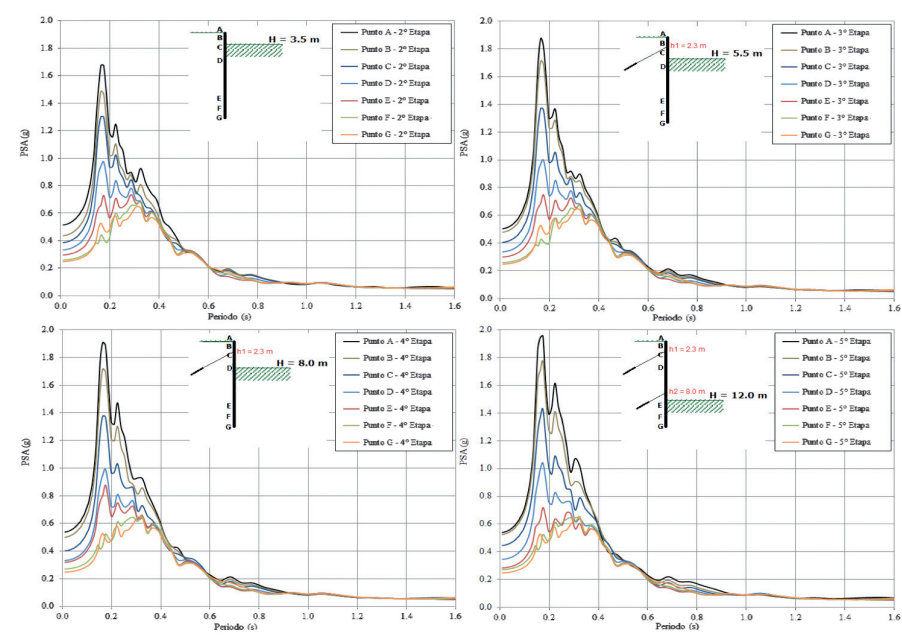

Figura 8: Respuesta espectral puntos de control sobre el muro para cuando la excavación es de 3.5, 5.5, 8 y $12 \mathrm{~m}$
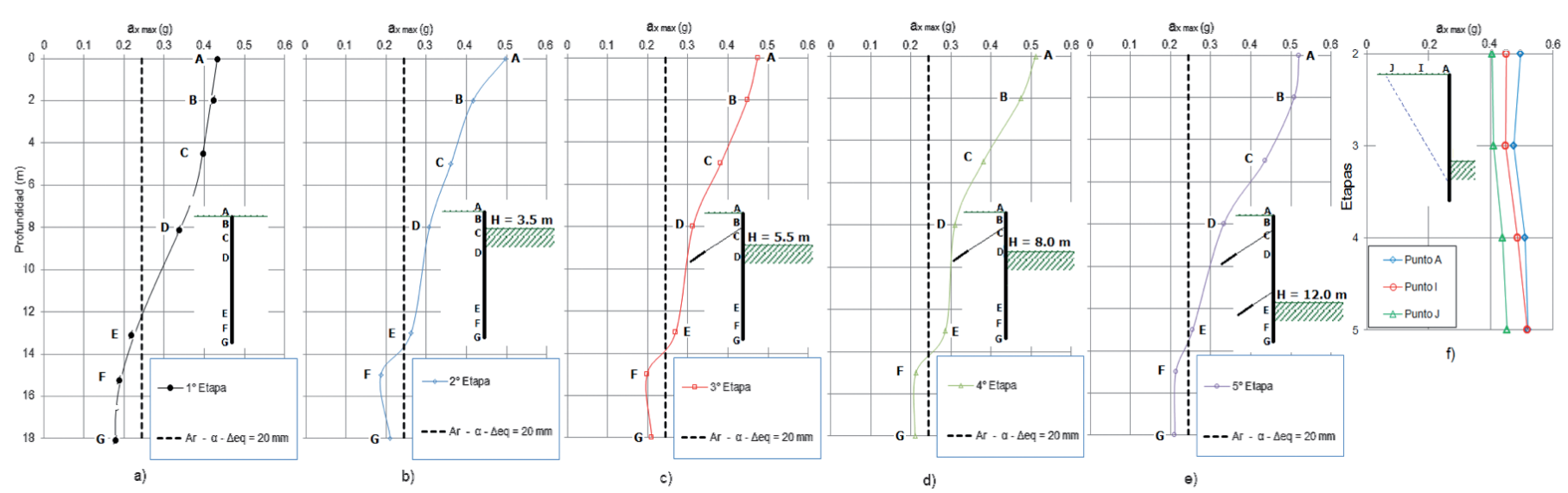

Figura 7: a) - e) PGA obtenidos en puntos de control para distintas etapas de excavación y f) PGA obtenidos en puntos de control detrás del muro 
En la Figura 9a se compara la respuesta espectral de los puntos $\mathrm{A}$ y $\mathrm{H}$, donde es posible apreciar que PSA es menor en el fondo de la excavación que en la parte superior del muro. La Figura 9b presenta los mismos resultados en mayor detalle, donde resulta aún más evidente la diferencia de la respuesta espectral llegando a ser incluso tres veces menor en el fondo de la excavación de $12 \mathrm{~m}$ apareciendo incluso un segundo periodo predominante. Por otro lado, la Figura 10 muestra la respuesta espectral de los puntos detrás del muro, donde no se observa un gran efecto después de excavar de $5.5 \mathrm{~m}$ a 8 y $12 \mathrm{~m}$. Es por ello que la atención está más puesta en los desplazamientos detrás del muro, lo cual se presenta a continuación.

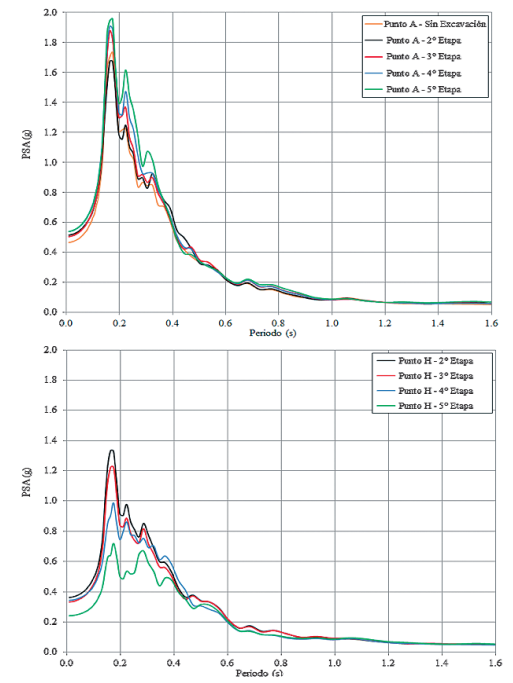

(a)

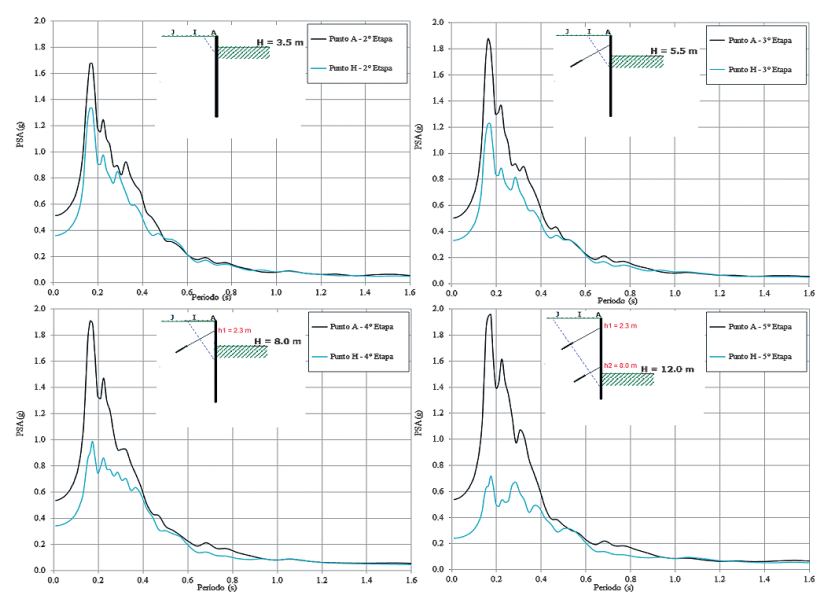

(b)

Figura 9: Comparación de respuesta espectral sobre el punto A del muro y el fondo de la excavación, a) punto A y $\mathrm{H}$ separados $\mathrm{y}$ b) juntos
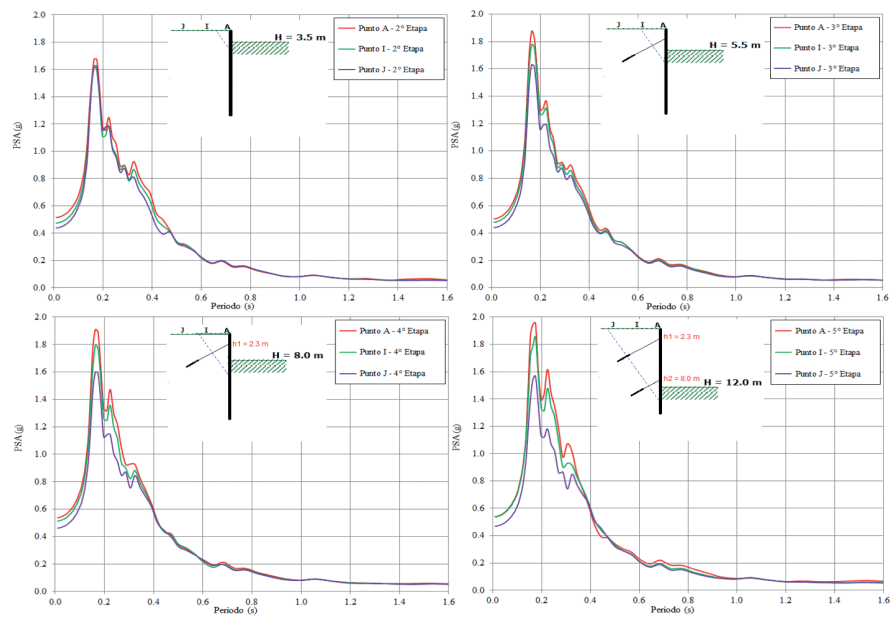

Figura 10: Respuesta espectral en la superficie detrás del muro

La respuesta sísmica del modelo 2D fue comparada con un modelo 1D realizado con el software DeepSoil (Hashash, 2012) mediante el método lineal equivalente. Los resultados indican que la distribución de pulsos de aceleración es similar y además los valores de periodos predominantes y pseudoaceleración PSA son cercanos, siendo para el modelo 2D: $0.17 \mathrm{~s}$ y $1.7 \mathrm{~g}$ y para el modelo 1D: $0.15 \mathrm{~s}$ y $2 \mathrm{~g}$. Esto demuestra que los módulos de rigidez utilizados para representar la propagación de ondas en el modelo 2D son satisfactorios. Por otro lado, los periodos fundamentales estimados a través del modelo $2 \mathrm{D}$ sin excavación fueron comparados con los valores medidos por Leyton et al. (2012) a través de microvibraciones en Concepción, siendo los valores estimados menores a los medidos. Lo anterior puede estar asociado al hecho de utilizar arena Bío Bío limpia para la modelación, en donde se ignora la presencia de los materiales finos que están presentes en la columna de suelo y que hacen más flexible la respuesta, aumentando el valor del periodo fundamental.

\section{Desplazamientos durante el movimiento sísmico}

Los siguientes resultados numéricos muestran que los desplazamientos están directamente relacionados con el número y distribución de pulsos de aceleración. La Figura 11 muestra la variación del desplazamiento horizontal $U_{\mathrm{x}}$, variación creciente casi lineal hasta los $20 \mathrm{~s}$, para luego variar con los pulsos. En la segunda etapa no existe mayor variación de $U_{\mathrm{x}}$ en los puntos de control, lo cual cambia para la tercera, cuarta y quinta etapa. Llama la 
atención que $U_{\mathrm{x}}$ alcanza siempre un valor cercano a $20 \mathrm{~cm}$, prácticamente los mismos resultados se obtuvieron para los puntos en la superficie detrás del muro (no mostrados). No olvidar que se está simulando la aplicación del mismo sismo en cada etapa de excavación sin cambiar ninguna de las propiedades del suelo ni del muro, solo la profundidad de la excavación.

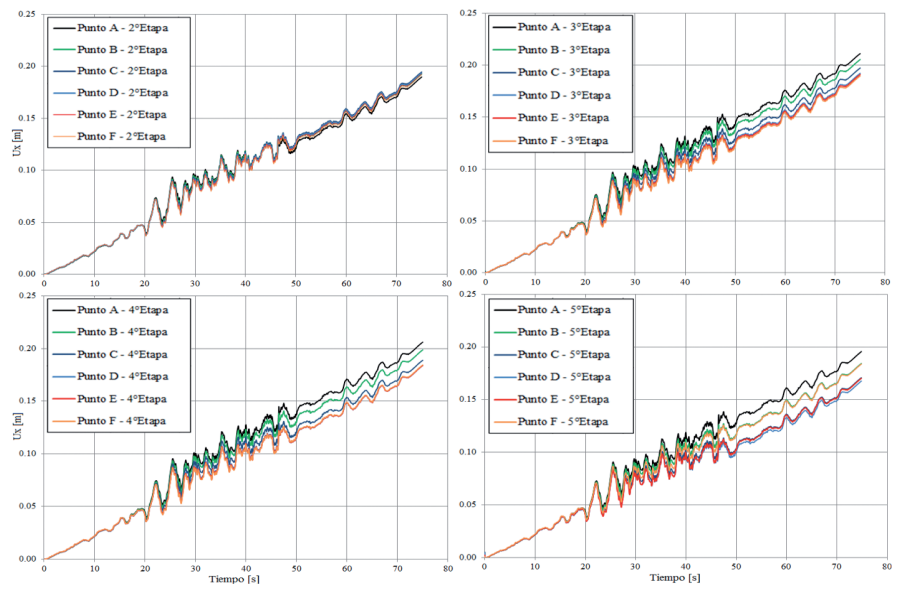

Figura 11: Desplazamientos horizontales $U_{\mathrm{x}}$ durante el sismo para puntos sobre el muro en diferentes etapas de excavación

La variación con el tiempo del desplazamiento vertical $U_{\mathrm{Y}}$ o asentamiento en la superficie detrás del muro es mostrada en la Figura 12. Nuevamente pareciera no haber diferencia de $U_{\mathrm{Y}}$ para las distintas etapas de excavación, alcanzándose un valor final constante a los $48 \mathrm{~s}$ de aproximadamente 6,7 y $8 \mathrm{~cm}$ de asentamiento en los puntos A, I y J, respectivamente. También llama la atención el mayor asentamiento en el punto más apartado. Estos aspectos serán nuevamente abordados en posteriores investigaciones.
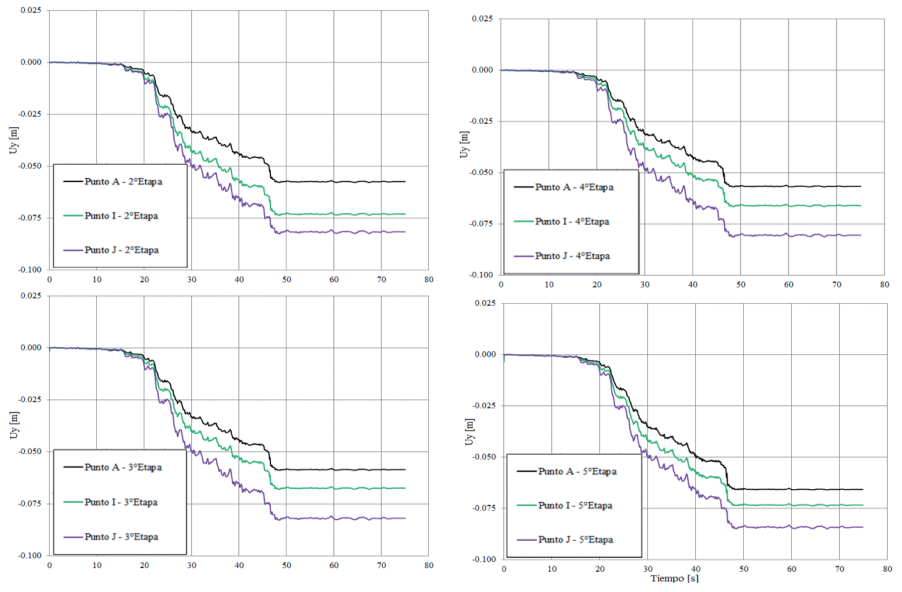

Figura 12: Desplazamientos verticales durante el sismo en puntos de control sobre la superficie detrás del muro

\section{Desplazamientos relativos}

La Figura 13 muestra que los desplazamientos horizontales relativos al punto $\mathrm{A}, \Delta U_{\mathrm{x}}$ para excavaciones menores a 5 $\mathrm{m}$ de profundidad presentan valores uniformes cercanos a $5 \mathrm{~mm}$, mientras que para profundidades de excavación superiores los valores son mayores a $20 \mathrm{~mm}$, superando los $20 \mathrm{~mm}$ recomendados por la NCh 3206 (2010). Además, el desplazamiento horizontal relativo al punto $\mathrm{A}$ al término del movimiento, es de al menos un 5\% mayor al registrado para la etapa anterior.

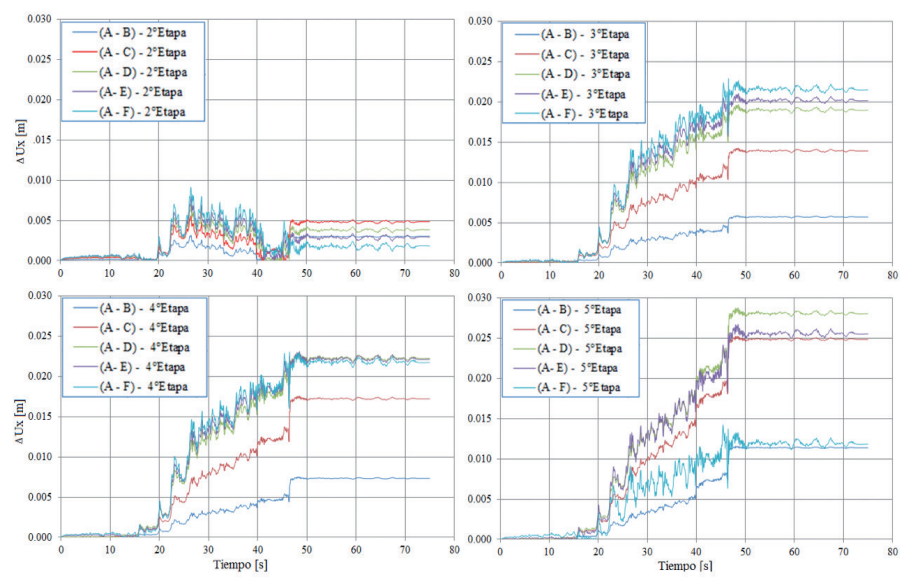

Figura 13: Desplazamientos horizontales relativos durante el sismo en puntos sobre el muro

La Figura 14 muestra el desplazamiento vertical relativo al punto $\mathrm{A}, \Delta U_{\mathrm{Y}} \mathrm{o}$ asentamiento diferencial de los puntos situados en la superficie detrás del muro, donde se alcanzan valores de hasta $25 \mathrm{~mm}$ para el punto J. También se muestra la diferencia entre el asentamiento vertical en el fondo de la excavación y el muro en el punto A, diferencia que llega a ser de hasta casi $50 \mathrm{~mm}$.
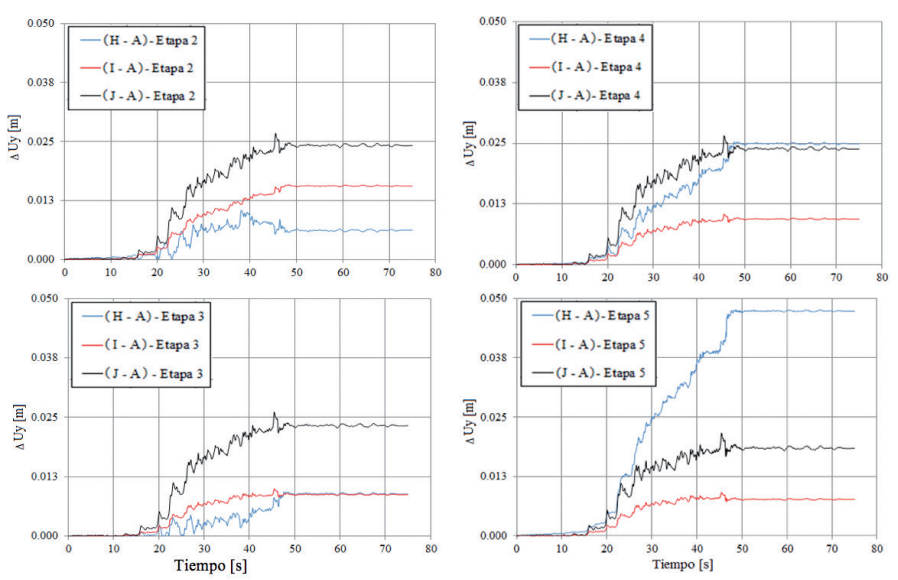

Figura 14: Desplazamientos verticales relativos al punto A durante el sismo para puntos de la superficie detrás del muro 


\section{Amplificación sísmica}

La amplificación sísmica en el punto A respecto a distintos puntos de control no mostró variaciones importantes al crecer la profundidad de excavación (ver Figuras $15 \mathrm{y}$ 16). Sin embargo, al estimar la amplificación sísmica con respecto a la base del depósito de arena se observa que para el modelo sin excavación los valores son similares para los puntos A, B y C, no así al considerar la excavación. En este caso la amplificación se incrementa de manera considerable alcanzándose amplificaciones de hasta 10 en el punto A, tal como se muestra en la Figura 17.

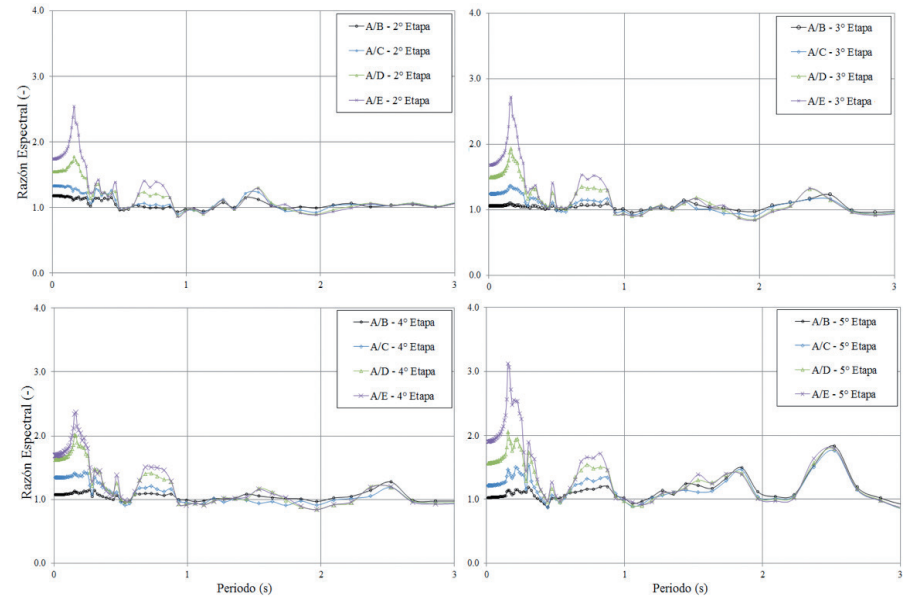

Figura 15: Razón espectral para puntos sobre el muro
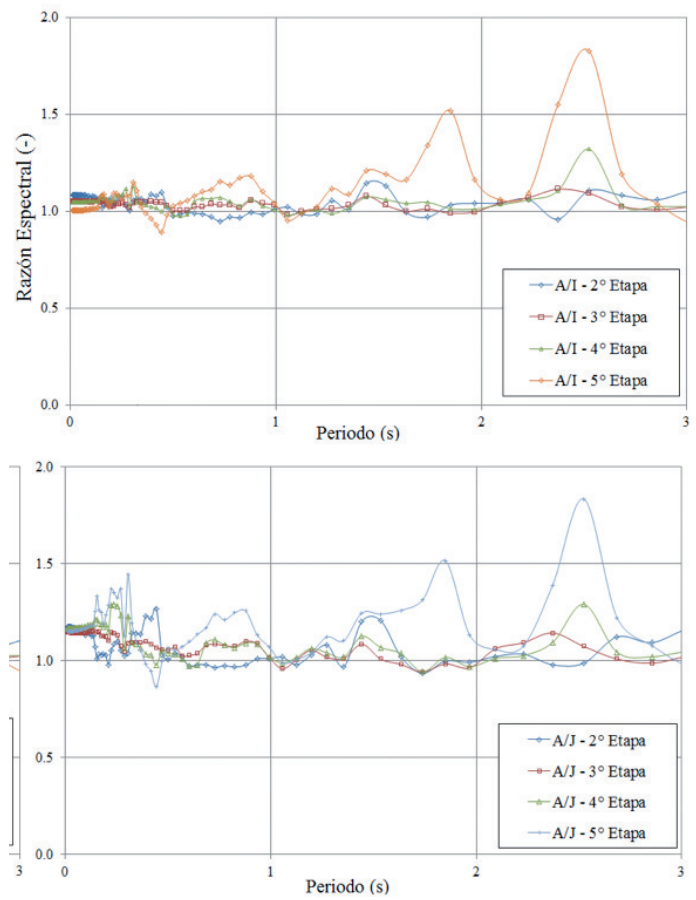

Figura 16: Razón espectral para puntos sobre la superficie detrás del muro
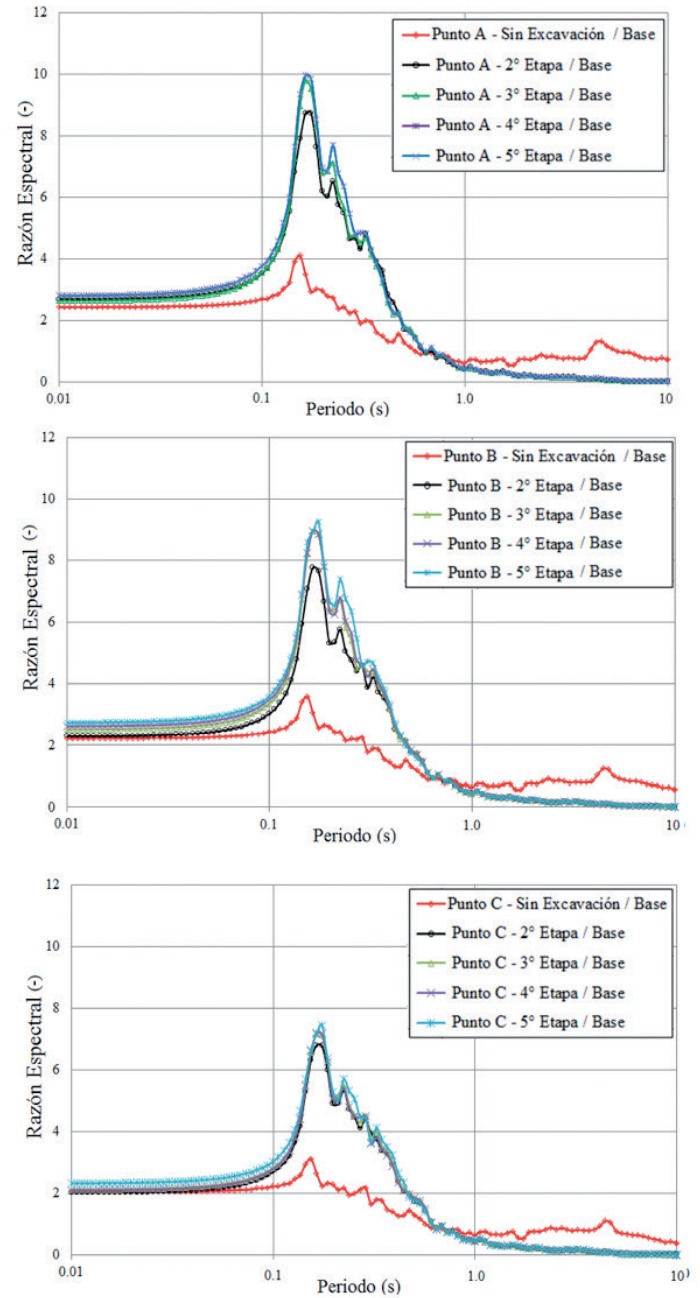

Figura 17: Razón espectral para los puntos A, B y C con respecto a la base

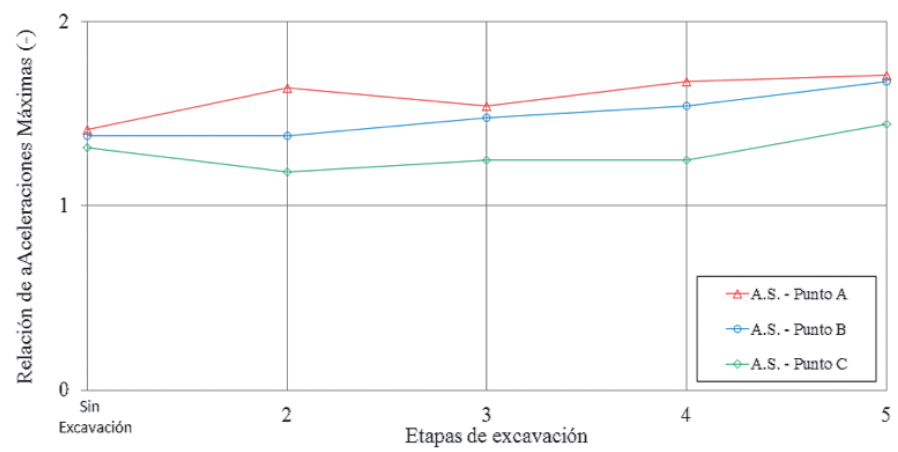

Figura 18: Relación de aceleraciones máximas respecto a las etapas de excavación (amplificación sísmica)

La Figura 18 entrega la amplificación sísmica como la razón de aceleraciones máximas respecto a la base, donde se aprecia que existe amplificación por sobre un factor de 1.2 hasta 1.6, y que el punto A es el que sufre una mayor amplificación sísmica. Cabe notar que si bien la amplificación aumenta levemente con la etapa de 
excavación para el punto $\mathrm{B}$, no es tan claro este aumento para los puntos A y C. Considerar que por ejemplo no se ha incluido en este análisis el efecto de los anclajes, los cuales pueden influir en la respuesta de la excavación dependiendo de la carga que estén tomando durante el sismo.

\section{Conclusiones}

Se utiliza un programa de elementos finitos comercial para determinar el efecto de las etapas constructivas de una excavación en la demanda sísmica sobre un muro Berlinés y el suelo circundante. Al comparar las demandas de aceleración superficial en excavaciones superiores a $10 \mathrm{~m}$, se observa un aumento de un $20 \%$ con respecto a un modelo sin excavación y además se excede la aceleración máxima $A_{\mathrm{o}}$ en un $30 \%$. Por otro lado, en superficie todas las etapas exceden a $A_{\mathrm{r}}$ en un $50 \%$. Se asocia este comportamiento al cambio de geometría superficial, que induce una reducción de rigidez y además perturba la llegada de las ondas de corte, favoreciendo la amplificación. La vecindad de la excavación se mostró alterada mostrando valores similares de aceleración. La respuesta espectral para cada etapa constructiva indica que no existe una variación importante del periodo fundamental en los primeros $12 \mathrm{~m}$ del muro y los valores de PSA se incrementan conforme mayor sea el volumen de material excavado. Además los desplazamientos horizontales absolutos para profundidades superiores a $5 \mathrm{~m}$ presentan valores de hasta $20 \mathrm{~cm}$ (mayores a lo recomendado por la NCh 3206, 2010). Finalmente, es importante recalcar la utilidad de estudiar el comportamiento sísmico de excavaciones a través de modelos bidimensionales que permiten estimar desplazamientos a diferencia de modelos de equilibrio límite. Futuras investigaciones consideran incluir el estudio de las fuerzas en los anclajes, estratos blandos de limo por ejemplo y sobrecargas de estructuras adyacentes a la excavación, además de modelar muros más rígidos como muros pantalla.

\section{Referencias}

Ayala, J. (2013). Estudio experimental de la propagación de ondas de corte en suelos granulares usando bender elements en el equipo edométrico. Tesis MSc, Universidad Católica de la Santísima Concepción
Gutiérrez, A. (1991). Las propiedades dinámicas de los suelos y su respuesta sísmica. Revista de Ingeniería, Universidad de Concepción, año 3, edición 1, 37-46

Hashash, Y.M.A. (2012). DEEPSOIL V 5.1, Tutorial and User Manual. University of Illinois at Urbana-Champaign

Kranz, E. (1953). Über die Verankerung von Spundwänden. Berlin: Ernst \& Sohn

Kuhlmeyer, R. and Lysmer, J. (1973). Finite element method accuracy for wave propagation problems. Journal of Soil Mechanics and Foundations Division 99 (SM5), 421 - 427

Leyton, F., Montalva, G. and Ramírez, P. (2012). A preliminary study of seismic microzonation of Concepción based on microtremors, geology and damages patterns. Obras y Proyectos $11,40-46$

Molina, D., Rivero, P.J. y Lobo, W. (2008). Respuesta espectral modificada por el nivel freático. Ciencia e Ingeniería 29, No. 3, 233-242,

Mozó, D., Oróstegui, P. y Villalobos, F. (2012). Proyecto de muros pantalla para el edificio Centro Plaza. VII Congreso Chileno de Geotecnia, Universidad de Concepción

Mozó, D. (2012). Análisis y diseño de muros pantalla en suelos arenosos. Proyecto de título de Ingeniero Civil, Universidad Católica de la Santísima Concepción

NCh 3206 (2010). Geotecnia - Excavaciones, entibaciones y socalzados. Instituto Nacional de Normalización INN, Santiago, Chile

Newmark, M.N. (1965). Effects of earthquakes on dams and embankments. Géotechnique 15(2), 139-160

Plaxis V8.2 (2010). Reference manual. Finite Element Code for Soil and Rock Analyses. Delft University of Tecnology, The Netherlands

Sharahi, M.J. (2010). The effect of geometry dimensions on the earthquake response on the finite element method. World Academy of Science, Engineering and Technology 4, 10-24

Schanz, T., Vermeer, P.A. and Bonnier, P.G. (1999). The hardening soil model: Formulation and verification. Beyond 2000 in Computational Geotechnics - 10 Years of Plaxis International. Balkema, Rotterdam 